\title{
Erratum to: Partial regularity at the first singular time for hypersurfaces evolving by mean curvature
}

\author{
Klaus Ecker ${ }^{1}$ (D)
}

Published online: 5 November 2015

(C) Springer-Verlag Berlin Heidelberg 2015

\section{Erratum to: Math. Ann. (2013) 356:217-240 DOI 10.1007/s00208-012-0853-6}

The purpose of this note is to inform the reader of a major error in the original article, namely in the proof of Theorem 1.8. I would like to point out that under the additional assumption of embeddedness of the solution the dimension estimates for $k$-convex hypersurfaces in Corollary 1.7 have been established by White [2] for $k=n$ and in [1] for all $1 \leq k \leq n$. I therefore believe that this statement is also correct in the case of immersed solutions but the proof may require different techniques to ours. I am also confident that the statement of Theorem 1.1 is correct for immersed hypersurfaces.

The error in the proof of Theorem 1.8 can be found in inequality (11) on page 13 of our paper. The centre of the ball should be $\frac{-y_{j}}{\lambda_{j}}$ rather than $-y_{j}$. The resulting ball may not contain the unit ball around the origin. Therefore, the validity of inequality (13) and all subsequent statements in the proof are unclear.

Since the proof of Theorem 1.1 uses Theorem 1.8 the validity of Theorem 1.1 is also unclear. The same holds for Corollary 1.2, Remark 1.3 as well as Corollaries 1.4 and 1.5. The curvature integral estimate for k-convex hypersurfaces with $1 \leq k \leq n-1$ is, however, correct. Corollary 1.7 relies on Corollary 1.2 though so its validity is again not clear.

I thank my colleague Dr. Theodora Bourni for pointing out this error to me.

The online version of the original article can be found under doi:10.1007/s00208-012-0853-6.

\footnotetext{
Klaus Ecker

kecker@zedat.fu-berlin.de

1 Berlin, Germany
} 


\section{References}

1. Cheeger, J., Haslhofer, R., Naber, A.: Quantitative stratification and the regularity of mean curvature flow. Geom. Funct. Anal. 23(3), 828-847 (2013)

2. White, B.: The size of the singular set in mean curvature flow of mean-convex sets. J. Am. Math. Soc. 13(3), 665-695 (2000) 